\title{
GENERALIZED STIELTJES POLYNOMIALS AND RATIONAL GAUSS-KRONROD QUADRATURE
}

\author{
M. BELLO HERNÁNDEZ, B. DE LA CALLE YSERN, AND G. LÓPEZ LAGOMASINO
}

\begin{abstract}
Generalized Stieltjes polynomials are introduced and their asymptotic properties outside the support of the measure are studied. As applications, we prove the convergence of sequences of interpolating rational functions, whose poles are partially fixed, to Markov functions and give an asymptotic estimate of the error of rational GaussKronrod quadrature formulae when functions which are analytic on some neighbourhood of the set of integration are considered.
\end{abstract}

\section{INTRODUCTION}

1.1. Stieltjes Polynomials. Let $\mu$ denote a finite positive Borel measure on the real line $\mathbb{R}$ whose compact support $S(\mu)$ contains infinitely many points. Let $\mu^{\prime}=d \mu / d x$ be the Radon-Nykodym derivative of $\mu$ with respect to the Lebesgue measure $d x$. Let $\left\{p_{n}\right\}_{n \in \mathbb{N}}$ be the sequence of orthonormal polynomials with respect to the measure $\mu$; that is, $p_{n}(z)=\kappa_{n} z^{n}+\cdots, \kappa_{n}>0$, and

$$
\int p_{m}(x) p_{k}(x) d \mu(x)=\delta_{k m}
$$

There exists a unique monic polynomial $S_{n}$ of degree $n$ which satisfies the orthogonality relations

$$
\int x^{k} S_{n}(x) p_{n-1}(x) d \mu(x)=0, \quad k=0,1, \ldots, n-1 .
$$

The polynomial $S_{n}$ is called the $n$th Stieltjes polynomial with respect to the measure $\mu$. We will refer to $s_{n}=\kappa_{n-1} S_{n}$ as the normalized $n$th Stieltjes polynomial. Much of the study on the polynomials $S_{n}$ has been directed into finding sufficient conditions guaranteeing that the zeros of $S_{n}$ have nice properties such as being simple and belonging to the set of integration. This is due to the fact that Stieltjes polynomials are used in Gauss-Kronrod quadrature rules (cf. [4], [7], and [1]; and the references therein). However, these properties are not satisfied in general (see, for instance, [8]). Until recently, little was known on the asymptotic behaviour of the Stieltjes polynomials outside the support of the measure. We say that the measure $\mu$ is regular and denote it by $\mu \in \mathbf{R e g}$ if one of the following two equivalent limit relations (see Theorem 3.1.1 in [12]) holds

$$
\lim _{n \rightarrow \infty} \kappa_{n}^{1 / n}=\frac{1}{\operatorname{cap} S(\mu)},
$$

1991 Mathematics Subject Classification. Primary: 42C05, 41A20, 65D32; Secondary: 30E10.

Key words and phrases. Stieltjes polynomials, varying measures, Gauss-Kronrod quadrature, rational quadrature, multipoint Padé-type approximation.

The work of M. Bello and B. de la Calle was partially supported by Dirección General de Enseñanza Superior (DGES) under grants BFM2000-0206-C04-03 and BFM2000-0206-C04-01 respectively. The work of G. López was partially supported by DGES under grant BFM2000-0206-C04-01 and by INTAS under grant $00-272$. 


$$
\lim _{n \rightarrow \infty}\left|p_{n}(z)\right|^{1 / n}=\exp \left\{g_{\Omega}(z, \infty)\right\},
$$

uniformly on compact subsets of $\mathbb{C} \backslash \operatorname{Co}(S(\mu))$, where $\operatorname{Co}(S(\mu))$ denotes the convex hull of $S(\mu)$, cap $S(\mu)$ stands for the logarithmic capacity of $S(\mu)$, and $g_{\Omega}(z, \infty)$ is the (generalized) Green function with singularity at infinity relative to the region $\Omega=\overline{\mathbb{C}} \backslash S(\mu)$ (cf. Section 1.2 and Appendix A.5 in [12] for the definition).

Regarding ratio asymptotics, E. A. Rakhmanov [9] proved that under the conditions $S(\mu)=[b-a, b+a]$ and $\mu^{\prime}>0$ almost everywhere on $[b-a, b+a]$

$$
\lim _{n \rightarrow \infty} \frac{p_{n+1}(z)}{p_{n}(z)}=\Psi\left(\frac{z-b}{a}\right),
$$

uniformly on compact subsets of $\mathbb{C} \backslash S(\mu)$, where $\Psi(z)=z+\sqrt{z^{2}-1}$. The square root is taken to be positive for $z>1$.

We also consider the Szegő class of measures. For simplicity in the notation, we restrict our attention to measures supported on $[-1,1]$. We say that $\mu \in \mathbf{S}$ if $S(\mu)=[-1,1]$ and $\log \mu^{\prime}(x) / \sqrt{1-x^{2}} \in L^{1}[-1,1]$. In this case

$$
\lim _{n \rightarrow \infty} \frac{p_{n}(z)}{\left[z+\sqrt{z^{2}-1}\right]^{n}}=\frac{1}{\sqrt{2 \pi}} S_{\mu}(\Psi(z)),
$$

uniformly on compact subsets of $\overline{\mathbb{C}} \backslash[-1,1]$, where

$$
S_{\mu}(z)=\exp \left\{\frac{1}{4 \pi} \int_{0}^{2 \pi} \log \left(\mu^{\prime}(\cos \theta)|\sin (\theta)|\right) \frac{e^{i \theta}+z}{e^{i \theta}-z} d \theta\right\}, \quad|z| \neq 1 .
$$

It is well known that $\mu \in \mathbf{S}$ implies that $\mu^{\prime}>0$ a.e. in $[-1,1]$ which, in turn, implies $\mu \in \mathbf{R e g}$ (see Theorem 4.1.1 in [12]). In [1] we proved the following theorem that is presented here in a simplified form for the case that $S(\mu)=[-1,1]$.

Theorem 1. The following assertions hold:

a) If $\mu \in \mathbf{R e g}$, the set of accumulation points of the zeros of $\left\{S_{n+1}\right\}_{n \in \mathbb{N}}$ is contained in $[-1,1]$ and

$$
\lim _{n \rightarrow \infty}\left|s_{n+1}(z)\right|^{1 / n}=\left|z+\sqrt{z^{2}-1}\right| .
$$

b) If $\mu^{\prime}>0$ a.e., then

$$
\lim _{n \rightarrow \infty} \frac{s_{n+1}(z)}{s_{n}(z)}=z+\sqrt{z^{2}-1}
$$

c) If $\mu \in \mathbf{S}$, then

$$
\lim _{n \rightarrow \infty} \frac{s_{n+1}(z)}{\left[z+\sqrt{z^{2}-1}\right]^{n}}=\sqrt{\frac{z^{2}-1}{2 \pi}} S_{\mu}(\Psi(z)) .
$$

The limits hold true uniformly on compact subsets of $\mathbb{C} \backslash[-1,1]$.

This result was used to obtain estimates on the rate of convergence of Gauss-Kronrod quadrature formulas and interpolating rational functions with partially prescribed poles for functions analytic on a certain neighbourhood of $[-1,1]$. 
1.2. Generalized Stieltjes Polynomials. Let $\left\{w_{n}\right\}_{n \in \mathbb{N}}$ be a sequence of monic polynomials with real coefficients such that, for each $n \in \mathbb{N}$ : $\operatorname{deg} w_{n}=i_{n}, 0 \leq i_{n} \leq 2 n+1$; and $w_{n}>0$ on $\operatorname{Co}(S(\mu))$. If $i_{n}<2 n+1$, let $w_{n, i}=\infty$ for $1 \leq i \leq 2 n+1-i_{n}$; if, $i_{n}>0$, then $\left\{w_{n, i}\right\}_{2 n+1-i_{n}+1 \leq i \leq 2 n+1}$, denotes the set of zeros of $w_{n}$. By $\mathbf{L}$ we denote the set of all limit points of $\left\{w_{n, i}\right\}_{1 \leq i \leq 2 n+1}$ as $n$ tends to infinity. In the sequel, we will assume that $\mathbf{L}$, which is a closed set, is contained in $\overline{\mathbb{C}} \backslash \operatorname{Co}(S(\mu))$. In fact, when the support of the measure is not an interval, it is necessary that $\mathbf{L}$ be contained in a more restricted set as we will see later. The positivity of $w_{n}$ on $\operatorname{Co}(S(\mu))$ is assumed for convenience in the normalization process but it would be sufficient that for each $n$ it preserves a constant sign on that set.

Set $d \mu_{n}=d \mu / w_{n}$. Obviously, for each $n \in \mathbb{N}$,

$$
\int_{S(\mu)} \frac{d \mu(x)}{w_{n}(x)}<+\infty
$$

therefore, we can construct the table of polynomials $\left\{p_{n, m}\right\}_{n, m \in \mathbb{N}}$, such that $p_{n, m}(z)=$ $\kappa_{n, m} z^{m}+\cdots, \kappa_{n, m}>0$, is the $m$ th orthonormal polynomial with respect to $\mu_{n}$. That is,

$$
\int_{S(\mu)} p_{n, k}(x) p_{n, m}(x) d \mu_{n}(x)=\delta_{k, m} .
$$

These polynomials are uniquely determined if we assume that the leading coefficients are positive. Unless otherwise specified, the set of integration is $S(\mu)$ in which case it will not be indicated.

Let $\left\{v_{n}\right\}_{n \in \mathbb{N}}$ be a sequence of monic polynomials with real coefficients such that $\operatorname{deg} v_{n}=$ $j_{n} \leq n+1$ and $v_{n}>0$ on $\operatorname{Co}(S(\mu))$. Let $v_{n, j}=\infty$ for $1 \leq j \leq n+1-j_{n}$; therefore, $\left\{v_{n, j}\right\}_{n+1-j_{n}+1 \leq j \leq n+1}$ denotes the set of zeros of $v_{n}$. We also assume that the set of all limit points of $\left\{v_{n, j}\right\}_{1 \leq j \leq n+1}$ as $n$ tends to infinity is contained in $\mathbf{L}$.

Let $\left\{S_{n, m}\right\}_{n, m \in \mathbb{N}}$ be a sequence of polynomials such that for each $n \in \mathbb{N}, S_{n, m}$ is defined as the monic polynomial of least degree verifying

$$
\int x^{k} S_{n, m}(x) p_{n, m-1}(x) \frac{d \mu_{n}(x)}{v_{n}(x)}=0, \quad k=0,1, \ldots, m-1 .
$$

Finding $S_{n, m}$ reduces to solving a system of $m$ homogeneous equations on $m+1$ unknowns. Thus a non-trivial solution always exists. We call $S_{n, m}$ the $m$ th Stieltjes polynomial with respect to the varying measure $d \mu_{n} / v_{n}$. The normalized Stieltjes polynomials $s_{n, m}$ is defined as $s_{n, m}=\kappa_{n, m-1} S_{n, m}$. In the case when $w_{n} \equiv v_{n} \equiv 1$ for all $n \in \mathbb{N}$, we regain the standard Stieltjes polynomials.

The object of this paper is to obtain similar results to those contained in Theorem 1 for generalized Stieltjes polynomials. This is done in Section 3. In Section 2 some preparatory work is carried out. Section 4 is dedicated to applications in two directions. Namely, the convergence of multipoint Padé-type approximation and the convergence of rational Gauss-Kronrod rules.

\section{Auxiliary Results}

2.1. Some Lemmas. The functions of second kind with respect to $\mu_{n}$ are given by

$$
g_{n, m}(z)=\int \frac{p_{n, m}(x)}{z-x} d \mu_{n}(x), \quad z \in \Omega=\overline{\mathbb{C}} \backslash S(\mu) .
$$


These functions are analytic in $\Omega$ and $g_{n, m}(\infty)=0$. Because of the orthogonality relations satisfied by $p_{n, m}$ with respect to the measure $\mu_{n}, z=\infty$ is a zero of $g_{n, m}$ of multiplicity $m+1$.

The next two lemmas are well known and easy to verify (see, for example, the proof of Theorem 6.1 .8 in [12]).

Lemma 1. We have

$$
g_{n, m}(z) p_{n, m}(z)=\int \frac{p_{n, m}^{2}(x)}{z-x} d \mu_{n}(x), \quad z \in \Omega .
$$

Lemma 2. Let $K$ be a compact subset of $\mathbb{C} \backslash \operatorname{Co}(S(\mu))$, then there exist positive constants $M_{1}, M_{2}$, independent of $n$ and $m$, satisfying

$$
M_{1} \leq\left|g_{n, m}(z) p_{n, m}(z)\right| \leq M_{2}, \quad z \in K .
$$

In particular, $g_{n, m}$ has no zeros on $\mathbb{C} \backslash \mathrm{Co}(S(\mu))$.

Lemma 3. If $m \geq \operatorname{deg} v_{n}$, then $\operatorname{deg} S_{n, m}=m$.

Proof. From the definition of $S_{n, m}$ it is immediate that $\operatorname{deg} S_{n, m} \leq m$. Let us suppose that $\operatorname{deg} S_{n, m} \leq m-1$. Due to the orthogonality relations satisfied by $p_{n, m-1}$ with respect to the measure $\mu_{n}$, we have

$$
\int \frac{S_{n, m}(z)-S_{n, m}(x)}{z-x} p_{n, m-1}(x) d \mu_{n}(x)=0 .
$$

Therefore

$$
\begin{aligned}
& S_{n, m}(z) g_{n, m-1}(z)=\int \frac{v_{n}(x) S_{n, m}(x)}{z-x} p_{n, m-1}(x) \frac{d \mu_{n}(x)}{v_{n}(x)} \\
& =v_{n}(z) \int \frac{S_{n, m}(x)}{z-x} p_{n, m-1}(x) \frac{d \mu_{n}(x)}{v_{n}(x)} .
\end{aligned}
$$

The second equality is a consequence of the orthogonality relations which $S_{n, m}$ satisfies with respect to $d \mu_{n} / v_{n}$ and the condition $m \geq \operatorname{deg} v_{n}$. Since $g_{n, m-1}$ has no zeros in $\mathbb{C} \backslash \operatorname{Co}(S(\mu))$, each zero of $v_{n}$ with multiplicity $k$ is a zero of $S_{n, m}$ with multiplicity at least $k$. Therefore, $\operatorname{deg} S_{n, m} \geq \operatorname{deg} v_{n}$.

On the other hand, we know that

$$
\int T(x) S_{n, m}(x) p_{n, m-1}(x) \frac{d \mu_{n}(x)}{v_{n}(x)}=0,
$$

for any polynomial $T$ of degree less than $m$. If we take in the relation above $T(x)=$ $v_{n}(x) x^{m-1-\operatorname{deg} S_{n, m}}$, we obtain

$$
\int x^{m-1-\operatorname{deg} S_{n, m}} S_{n, m}(x) p_{n, m-1}(x) d \mu_{n}(x)=0 .
$$

Notice that $\operatorname{deg}\left(x^{m-1-\operatorname{deg} S_{n, m}} S_{n, m}(x)\right)=m-1$ and $p_{n, m-1}$ is orthogonal to all polynomials of degree less than $m-1$ with respect to $\mu_{n}$. Therefore, $p_{n, m-1}$ is orthogonal to itself. This contradiction indicates that $\operatorname{deg} S_{n, m}=m$. 
The next lemma plays a key role in our subsequent arguments. Its proof is basically the same as that of Lemma 2 in [1] although we include it for the sake of completeness.

Lemma 4. If $m \geq \operatorname{deg} v_{n}$, for all $z \in \overline{\mathbb{C}} \backslash \operatorname{Co}(S(\mu))$ we have

$$
\frac{s_{n, m}(z)-1 / g_{n, m-1}(z)}{v_{n}(z)}=\frac{1}{g_{n, m-1}(z)} \int \frac{s_{n, m}(x)}{z-x} p_{n, m-1}(x) \frac{d \mu_{n}(x)}{v_{n}(x)}
$$

and

$$
s_{n, m}(z) g_{n, m-1}(z)=1+\frac{v_{n}(z) g_{n, m-1}(z)}{2 \pi i} \int_{\gamma} \frac{d \zeta}{v_{n}(\zeta) g_{n, m-1}(\zeta)(\zeta-z)}
$$

where $\gamma$ is any positively oriented close smooth curve which surrounds the set $\operatorname{Co}(S(\mu))$ such that $\mathbf{L}$ and $z$ are contained in the unbounded component of $\overline{\mathbb{C}} \backslash \gamma$.

PROOF. From the orthogonality relations of $p_{n, m-1}$ with respect to the measure $\mu_{n}$, we obtain

$$
\begin{gathered}
\int \frac{S_{n, m}(z)-S_{n, m}(x)}{z-x} p_{n, m-1}(x) d \mu_{n}(x)=\int \frac{z^{m}-x^{m}}{z-x} p_{n, m-1}(x) d \mu_{n}(x) \\
=\int x^{m-1} p_{n, m-1}(z) d \mu_{n}(x)=\frac{1}{\kappa_{n, m-1}} .
\end{gathered}
$$

Rewriting this equation and using the orthogonality of $S_{n, m}$ with respect to $d \mu_{n} / v_{n}$, we find that

$$
\begin{aligned}
g_{n, m-1}(z) s_{n, m}(z) & =1+\int \frac{s_{n, m}(x)}{z-x} p_{n, m-1}(x) d \mu_{n}(x) \\
& =1+\int \frac{v_{n}(x) s_{n, m}(x)}{z-x} p_{n, m-1}(x) \frac{d \mu_{n}(x)}{v_{n}(x)} \\
& =1+v_{n}(z) \int \frac{s_{n, m}(x)}{z-x} p_{n, m-1}(x) \frac{d \mu_{n}(x)}{v_{n}(x)}
\end{aligned}
$$

The first formula of the lemma is equivalent to this expression. From (1) and the orthogonality properties of $p_{n, m-1}$ and $s_{n, m}$, we have that

$$
\frac{s_{n, m}(z)-1 / g_{n, m-1}(z)}{v_{n}(z)}=\frac{\int \frac{x s_{n, m}(x)}{z-x} p_{n, m-1}^{2}(x) \frac{d \mu_{n}(x)}{v_{n}(x)}}{\int \frac{p_{n, m-1}^{2}(x)}{1-x / z} d \mu_{n}(x)} .
$$


Therefore, this function is analytic in $\overline{\mathbb{C}} \backslash \operatorname{Co}(S(\mu))$ and has a zero of order at least 1 at infinity. Using Cauchy's integral formula with a curve $\gamma$ as indicated above, we obtain

$$
\begin{aligned}
\frac{s_{n, m}(z)-1 / g_{n, m-1}(z)}{v_{n}(z)} & =\frac{1}{2 \pi i} \int_{\gamma} \frac{s_{n, m}(\zeta)-1 / g_{n, m-1}(\zeta)}{v_{n}(\zeta)(z-\zeta)} d \zeta \\
& =\frac{1}{2 \pi i} \int_{\gamma} \frac{d \zeta}{v_{n}(\zeta) g_{n, m-1}(\zeta)(\zeta-z)}
\end{aligned}
$$

Rewriting this formula conveniently, we obtain (2). The proof is completed.

Notice that $g_{n, m-1}(z)$ may have zeros in $\operatorname{Co}(S(\mu)) \backslash S(\mu)$; therefore, $1 / g_{n, m-1}(z)$ can have poles in this set. This is the only reason why, in general, we cannot take a curve $\gamma$ closer to $S(\mu)$ than the way indicated in Lemma 4.

2.2. Potential Theory. Throughout this section, we will assume that $\mathbb{C} \backslash S(\mu)$ is a regular domain with respect to the Dirichlet problem. In reference to this condition, for simplicity, we will say that $S(\mu)$ is regular. The regularity of $S(\mu)$ implies that cap $S(\mu)>0$.

Let $w$ be a positive continuous function on $S(\mu)$. Set $f(z)=-\log w(z)$. It is well known (see [11], Sections I.1 and I.3) that among all probability measures $\sigma$ with support in $S(\mu)$ there exists a unique probability measure $\mu_{w}$ with support in $S(\mu)$, called the extremal or equilibrium measure associated with $w$, minimizing the weighted energy

$$
I_{w}(\sigma)=\iint\left(\log \frac{1}{|z-t|}+f(z)+f(t)\right) d \sigma(z) d \sigma(t) .
$$

Let $P\left(\mu_{w} ; z\right)=-\int \log |z-t| d \mu_{w}(t)$ be the potential of this extremal measure and $S_{w} \subset$ $S(\mu)$ its support. Under these conditions there exists a constant $F_{w}$, called equilibrium constant, such that

$$
\begin{aligned}
& P\left(\mu_{w} ; z\right)+f(z) \geq F_{w}, \quad z \in S(\mu), \\
& P\left(\mu_{w} ; z\right)+f(z)=F_{w}, \quad z \in S_{w} .
\end{aligned}
$$

Due to $(3), \mu_{w}$ is also called the equilibrium measure in presence of the external field $f$.

For regular measures, the $n$th root asymptotic behaviour of orthogonal polynomials with respect to varying measures is characterized by the equilibrium measure in presence of the external field induced by the varying part of the measure. The corresponding result has been proved in various degrees of generality by different authors. We state it as it appears in [12], Theorem 6.5.1, where a proof and more references may be found.

Let $\mu \in \mathbf{R e g}$ and let $\left\{w_{n}\right\}_{n \in \mathbb{N}}$ be as defined in Section 1.2. Assume that $\left(w_{n}\right)^{-1 / n}$ converges uniformly to $w^{2}$ on $S(\mu)$, where $w$ is a positive continuous function on $S(\mu)$. Then

$$
\lim _{n \rightarrow \infty}\left(\kappa_{n, n}\right)^{1 / n}=e^{F_{w}}
$$

and

$$
\lim _{n \rightarrow \infty}\left|p_{n, n}(z)\right|^{1 / n}=e^{F_{w}-P\left(\mu_{w} ; z\right)}
$$

uniformly on each compact subset of $\mathbb{C} \backslash \operatorname{Co}(S(\mu))$. 
Notice that for each $k \in \mathbb{Z}$ fixed, (4) and (5) remain in force for the sequences $\left\{\kappa_{n, n+k}\right\}$ and $\left\{p_{n, n+k}\right\}$ respectively, with the same expression in the right hand. In fact, using the same result, in order to find the new limits, we would have to find $\lim _{n}\left(w_{n}\right)^{-1 / n+k}=$ $\lim _{n}\left(w_{n}\right)^{-1 / n}=w^{2}$. Therefore, we get the same external field.

Let $\rho_{n}$ and $\rho$ be finite Borel measures on $\overline{\mathbb{C}}$. By $\rho_{n} \stackrel{*}{\longrightarrow} \rho, n \rightarrow \infty$, we denote the weak ${ }^{*}$ convergence of $\rho_{n}$ to $\rho$ as $n$ tends to infinity. This means that for every continuous function $f$ on $\overline{\mathbb{C}}$

$$
\lim _{n \rightarrow \infty} \int f(x) d \rho_{n}(x)=\int f(x) d \rho(x) .
$$

For a given polynomial $T$, we denote by $\Lambda_{T}$ the normalized zero counting measure of $T$. That is

$$
\Lambda_{T}=\frac{1}{\operatorname{deg} T} \sum_{\xi: T(\xi)=0} \delta_{\xi} .
$$

The sum is taken over all the zeros of $T$ and $\delta_{\xi}$ denotes the Dirac measure concentrated at $\xi$.

In the sequel, for each $n$, it is considered that $\operatorname{deg} w_{n}=2 n+1$ and $\operatorname{deg} v_{n}=n+1$, assigning to these polynomials $2 n+1-i_{n}$ and $n+1-j_{n}$ "zeros" at infinity respectively in case that either $i_{n}<2 n+1$ or $j_{n}<n+1$ (for the meaning of $i_{n}$ and $j_{n}$ return to Section 1.2 where the polynomials $w_{n}$ and $v_{n}$ were introduced). It is said that the sequence of polynomials $\left\{w_{n}\right\}_{n \in \mathbb{N}}$ (analogously for $\left\{v_{n}\right\}_{n \in \mathbb{N}}$ ) has $\nu$ as its zero asymptotic distribution if

$$
\Lambda_{w_{n}} \stackrel{*}{\longrightarrow} \nu, \quad n \rightarrow \infty .
$$

If $\left\{w_{n}\right\}_{n \in \mathbb{N}}$ has zero asymptotic distribution $\nu$, it is easy to see that $\left(w_{n}\right)^{-1 / n}$ uniformly converges to $e^{2 P(\nu ; .)}$ on $S(\mu)$, where $P(\nu ; \cdot)$ is the potential of the probability measure $\nu$. Then, the asymptotic behaviour of the polynomials $p_{n, n}$ may be expressed in terms of the equilibrium measure $\mu_{w}$ in the presence of the external field $-P(\nu ; \cdot)$. Since the support of $\nu$ is contained in $\mathbf{L} \subset \overline{\mathbb{C}} \backslash \operatorname{Co}(S(\mu))$, it is well known that $\mu_{w}$ is the balayage of $\nu$ onto $S(\mu)$. Therefore, $S_{w} \equiv S(\mu)$ (see, for instance [11], Chapter IV, Theorem 1.10) and

$$
P\left(\mu_{w} ; z\right)-P(\nu ; z)=F_{w}, \quad z \in S(\mu) .
$$

If $S(\mu)$ is made up by several intervals, the measure $\mu_{w}$ is absolutely continuous with respect to the Lebesgue measure $d x$ and

$$
\frac{\partial\left(\mu_{w}\right)}{\partial x}(x)=\frac{1}{2 \pi} \int_{\mathbf{L}}\left(\frac{\partial g_{\Omega}}{\partial n_{+}}(x ; a)+\frac{\partial g_{\Omega}}{\partial n_{-}}(x ; a)\right) d \nu(a),
$$

where $g_{\Omega}(\cdot ; a)$ is the Green's function of $\Omega$ with singularity at the point $a$ and $n_{ \pm}$stands for the two normals to the real line. We can make the above formula more precise if, additionally, we suppose that $S(\mu)=[-1,1]$ and $\nu=\lambda \delta_{\infty}+(1-\lambda) \tilde{\nu}$, where $\tilde{\nu}$ is a measure supported on $\mathbb{R} \backslash[-1,1]$. Then (cf. [11], pp. 118-122), for each $x \in[-1,1]$, we have

$$
\frac{\partial\left(\mu_{w}\right)}{\partial x}(x)=\frac{\lambda}{\pi} \frac{1}{\sqrt{1-x^{2}}}+\frac{1-\lambda}{\pi} \int \frac{\left|\sqrt{a^{2}-1}\right|}{|x-a| \sqrt{1-x^{2}}} d \nu(a) .
$$

It is also known (see Theorem 5.1, Chapter II in [11]) that

$$
G_{\Omega}(\nu ; z)=F_{w}-P\left(\mu_{w} ; z\right)+P(\nu ; z), \quad z \in \Omega,
$$


where $G_{\Omega}(\nu ; \cdot)$ is the Green potential of the measure $\nu$ in $\Omega$; that is,

Set

$$
G_{\Omega}(\nu ; z)=\int_{\mathbf{L}} g_{\Omega}(z ; \zeta) d \nu(\zeta), \quad z \in \Omega
$$

$$
E=\left\{z \in \mathbb{C}: G_{\Omega}(\nu ; z) \leq \max _{\zeta \in \operatorname{Co}(S(\mu))} G_{\Omega}(\nu ; \zeta)\right\} .
$$

From some examples in [1], it follows that there is no hope of obtaining asymptotics of the (generalized) Stieltjes polynomials inside the set $E$ for the whole class of regular measures.

\section{Asymptotics}

3.1. Weak Asymptotics. In the sequel, we will suppose that $\mathbf{L} \subset \overline{\mathbb{C}} \backslash E$. Without loss of generality, we may assume that $\mathbf{L}$ is a compact subset of $\mathbb{C} \backslash E$. The reduction to this case may be achieved by means of a Möbius transformation of the variable in the initial problem, which transforms $S(\mu)$ into another compact subset of $\mathbb{R}$ and $\mathbf{L} \subset \overline{\mathbb{C}} \backslash E$ into a compact subset contained in $\mathbb{C} \backslash \widetilde{E}$, where $\widetilde{E}$ is the image of $E$ by the Möbius transformation. This assumption implies, in particular, that for each $n$ the degrees of $v_{n}$ and $w_{n}$ are really $n+1$ and $2 n+1$ respectively, and liberates our arguments from the special treatment which otherwise we would have to give to neighbourhoods of infinity. We use this assumption in the proofs but state the results for an arbitrary $\mathbf{L}$ contained in $\overline{\mathbb{C}} \backslash E$.

Theorem 2. Suppose that the sequences of polynomials $\left\{w_{n}\right\}$ and $\left\{v_{n}\right\}$ have $\nu$ as their zero asymptotic distribution. Let $k$ be a fixed integer, $k \geq 0$. If $S(\mu)$ is regular and $\mu \in \mathbf{R e g}$ then

$$
\begin{aligned}
& \limsup _{n \rightarrow \infty}\left\|s_{n, n+k+1} g_{n, n+k}-1\right\|_{K}^{1 / n} \leq \\
& \left\|\exp \left\{-G_{\Omega}(\nu ; z)\right\}\right\|_{K}\left\|\exp \left\{G_{\Omega}(\nu ; z)\right\}\right\|_{\operatorname{Co}(S(\mu))},
\end{aligned}
$$

where $K$ is any compact subset of $\overline{\mathbb{C}} \backslash E$.

Proof. Fix a compact set $K \subset \mathbb{C} \backslash E$. Let $V$ be a neighbourhood of $\mathbf{L}$ such that $\bar{V} \subset$ $\mathbb{C} \backslash E$. Take $n_{0}$ sufficiently large so that for $n \geq n_{0}$ all the zeros of $v_{n}$ lie in $V$. Fix $r>\left\|G_{\Omega}(\nu ; \zeta)\right\|_{\mathrm{Co}(S(\mu))}$ so that $\bar{V} \cup K$ lies in the unbounded component of $\mathbb{C} \backslash \gamma_{r}$, where $\gamma_{r}=\left\{\zeta \in \mathbb{C}: G_{\Omega}(\nu ; \zeta)=r\right\}$. From (2), applied integrating over $\gamma_{r}$, we have that

$$
\left\|s_{n, n+k+1} g_{n, n+k}-1\right\|_{K} \leq C \frac{\left\|v_{n} g_{n, n+k}\right\|_{K}}{\inf _{\zeta \in \gamma_{r}}\left|v_{n}(\zeta) g_{n, n+k}(\zeta)\right|}
$$

where $C$ is a positive constant depending on the length of $\gamma_{r}$ and the distance between $\gamma_{r}$ and $K$, but not on $n \geq n_{0}$. Therefore,

$$
\limsup _{n \rightarrow \infty}\left\|s_{n, n+k+1} g_{n, n+k}-1\right\|_{K}^{1 / n} \leq \frac{\limsup _{n \rightarrow \infty}\left\|v_{n} g_{n, n+k}\right\|_{K}^{1 / n}}{\lim _{n \rightarrow \infty} \inf _{\zeta \in \gamma_{r}}\left|v_{n}(\zeta) g_{n, n+k}(\zeta)\right|^{1 / n}} .
$$

By Lemma 2 and (5) (taking into account the remark made on the sentence following (5)), we have that

$$
\lim _{n \rightarrow \infty}\left|g_{n, n+k}(z)\right|^{1 / n}=e^{P\left(\mu_{w} ; z\right)-F_{w}}
$$


uniformly on compact subsets of $\mathbb{C} \backslash \operatorname{Co}(S(\mu))$. Since $\nu$ is the zero asymptotic distribution of $\left\{v_{n}\right\}_{n \in \mathbb{N}}$, then

$$
\lim _{n \rightarrow \infty}\left|v_{n}(z)\right|^{1 / n}=e^{-P(\nu ; z)},
$$

uniformly on compact subsets of $\mathbb{C} \backslash \mathbf{L}$, and using the Principle of Descent (see [11], Chapter I, Theorem 6.8), we have that

$$
\limsup _{n \rightarrow \infty}\left|v_{n}(z)\right|^{1 / n} \leq e^{-P(\nu ; z)},
$$

uniformly on compact subsets of $\mathbb{C}$. From (9), (10), and (11), taking account of (6), it follows that

$$
\limsup _{n \rightarrow \infty}\left\|v_{n} g_{n, n+k}\right\|_{K}^{1 / n} \leq\left\|\exp \left\{-G_{\Omega}(\nu ; z)\right\}\right\|_{K}
$$

and

$$
\lim _{n \rightarrow \infty} \inf _{\zeta \in \gamma_{r}}\left|v_{n}(\zeta) g_{n, n+k}(\zeta)\right|^{1 / n}=e^{-r} .
$$

Relations (12) and (13) together with (8) give

$$
\limsup _{n \rightarrow \infty}\left\|s_{n, n+k+1} g_{n, n+k}-1\right\|_{K}^{1 / n} \leq\left\|\exp \left\{-G_{\Omega}(\nu ; z)\right\}\right\|_{K} e^{r} .
$$

The left hand of this inequality does not depend on $r$; therefore, we can make $r$ tend to $\left\|G_{\Omega}(\nu ; \zeta)\right\|_{\mathrm{Co}(S(\mu))}$ obtainig (7) for compact subsets of $\mathbb{C} \backslash E$. The function under the norm sign on the left hand of $(7)$ is analytic on $\overline{\mathbb{C}} \backslash S(\mu)$ and, in particular, on $\overline{\mathbb{C}} \backslash E$; therefore, by use of the Maximum Principle the result is easily extended to compact subsets of $\overline{\mathbb{C}} \backslash E$. With this we conclude the proof.

Corollary 1. Under the hypothesis of Theorem 2, we have that

$$
\lim _{n \rightarrow \infty} s_{n, n+k+1}(z) g_{n, n+k}(z)=1,
$$

uniformly on compact subsets of $\overline{\mathbb{C}} \backslash E$. The set of accumulation points of the zeros of $\left\{S_{n, n+k+1}\right\}_{n \in \mathbb{N}}$ is contained in E. Also

$$
\lim _{n \rightarrow \infty}\left|s_{n, n+k+1}\right|^{1 / n}(z)=e^{F_{w}-P\left(\mu_{w} ; z\right)},
$$

uniformly on each compact subset of $\mathbb{C} \backslash E$.

Proof. Since $\left\|\exp \left\{-G_{\Omega}(\nu ; z)\right\}\right\|_{K}\left\|\exp \left\{G_{\Omega}(\nu ; z)\right\}\right\|_{\operatorname{Co}(S(\mu))}<1$ due to the subharmonicity of $-G_{\Omega}(\nu ; z)$ on $\overline{\mathbb{C}} \backslash E$, relation (14) follows immediately from (7). The statement concerning the zeros of $\left\{S_{n, n+k+1}\right\}_{n \in \mathbb{N}}$ is a direct consequence of (14) and Hurwitz's Theorem since the function 1 has no zeros on $\overline{\mathbb{C}} \backslash E$. Finally, (14) and (9) render (15).

Now, let us say some words about the case when $S(\mu)$ is an interval. Since $g_{\Omega}(z ; \zeta) \equiv 0$ on $S(\mu)$ we have that $G_{\Omega}(\nu ; z) \equiv 0$ on $S(\mu)$. Additionally, $G_{\Omega}(\nu ; z)>0$ on $\Omega$ because of its superharmonicity. Therefore, in this particular case, $E \equiv S(\mu)$ and Theorem 2 and Corollary 1 hold true on all $\overline{\mathbb{C}} \backslash S(\mu)$. In the following theorem, we give more information about the zeros of generalized Stieltjes polynomials. 
Theorem 3. Let $S(\mu)$ be an interval. Suppose that the sequences of polynomials $\left\{w_{n}\right\}_{n \in \mathbb{N}}$ and $\left\{v_{n}\right\}_{n \in \mathbb{N}}$ have $\nu$ as their zero asymptotic distribution and $\mu \in \mathbf{R e g}$. Then

$$
\Lambda_{S_{n, n+k+1}} \stackrel{*}{\longrightarrow} \mu_{w}, \quad n \rightarrow \infty .
$$

Proof. Set $\Lambda_{S_{n, n+k+1}}=\Lambda_{n}$ and denote the potential of $\Lambda_{n}$ by $U_{n}$. All the measures $\Lambda_{n}$ are probability measures. Let $\Delta \subset \mathbb{N}$ be a subsequence of indices such that

$$
\Lambda_{n} \stackrel{*}{\longrightarrow} \Lambda, \quad n \in \Delta, \quad n \rightarrow \infty .
$$

It is sufficient to prove that $\Lambda=\mu_{w}$ for any such sequence $\Delta$ of indexes. Corollary 1 indicates that the support of $\Lambda$ is contained in the set $S(\mu)$. In addition to this, we know, due to Lemma 3 , that $\operatorname{deg} S_{n, n+k+1}=n+k+1$.

Taking (15) and (4) into account, we have that

$$
\lim _{n \in \Delta} U_{n}(z)=\lim _{n \in \Delta} \frac{-1}{n+k+1} \log \left|S_{n, n+k+1}\right|=P\left(\mu_{w}, z\right), \quad z \in \mathbb{C} \backslash S(\mu) .
$$

On the other hand, from (16) one obtains

$$
\lim _{n \in \Delta} U_{n}(z)=U_{\Lambda}(z), \quad z \in \mathbb{C} \backslash S(\mu),
$$

where $U_{\Lambda}$ is the potential of the measure $\Lambda$. Thus, $U_{\Lambda}(z)=P\left(\mu_{w}, z\right)$ except at most on a set of Lebesgue measure zero in the complex plane; therefore, from Theorem 3.7.4 in [10], we obtain that $\Lambda=\mu_{w}$ as we wanted to prove.

3.2. Ratio Asymptotics. Unless otherwise stated, in this section and the next, for the sake of simplicity, we will consider that the support of the measure $\mu$ is the interval $[-1,1]$, but all the results can be restated if we substitute $[-1,1]$ by any compact interval $I$ of $\mathbb{R}$. As usual, the reduction to the interval $[-1,1]$ is attained by means of an affine change of variables.

It is known that if $\mu^{\prime}>0$ a.e. on $[-1,1]$ and $\mathbf{L} \cap[-1,1]=\emptyset$, then for each fixed $k \in \mathbb{N}$

$$
\lim _{n \rightarrow \infty} \frac{p_{n, n+k+1}(z)}{p_{n, n+k}(z)}=z+\sqrt{z^{2}-1}
$$

uniformly on each compact subset of $\mathbb{C} \backslash[-1,1]$. Moreover, for every $m \in \mathbb{N}$ and every bounded Borel-measurable function $f$ on $[-1,1]$, we have

$$
\lim _{n \rightarrow \infty} \int_{-1}^{1} f(x) p_{n, n+k}(x) p_{n, n+k+m}(x) d \mu_{n}(x)=\frac{1}{\pi} \int_{-1}^{1} f(x) T_{m}(x) \frac{d x}{\sqrt{1-x^{2}}},
$$

where $T_{m}$ denotes the $m$ th Chebyshev polynomial, i.e., $T_{m}(\cos \theta)=\cos m \theta$. In particular

$$
\lim _{n \rightarrow \infty} g_{n, n+k}(z) p_{n, n+k}(z)=\frac{1}{\pi} \int_{-1}^{1} \frac{d x}{(z-x) \sqrt{1-x^{2}}}=\frac{1}{\sqrt{z^{2}-1}},
$$

uniformly on each compact subset of $\overline{\mathbb{C}} \backslash[-1,1]$, where the square root is taken so that $\sqrt{z^{2}-1}>0$ for $z>1$. The proof of these results may be found in [3].

With the aid of the theorems of the previous section, we can derive ratio asymptotics for sequences of generalized Stieltjes polynomials. 
Theorem 4. Suppose that the sequences of polynomials $\left\{w_{n}\right\}$ and $\left\{v_{n}\right\}$ have the same zero asymptotic distribution. Let $k$ be a fixed integer, $k \geq 0$. If $S(\mu)=[-1,1]$ and $\mu^{\prime}>0$ a.e. on $[-1,1]$, then

$$
\lim _{n \rightarrow \infty} \frac{s_{n, n+k+1}(z)}{s_{n, n+k}(z)}=z+\sqrt{z^{2}-1}
$$

and

$$
\lim _{n \rightarrow \infty} \frac{p_{n, n+k}(z)}{s_{n, n+k+1}(z)}=\frac{1}{\sqrt{z^{2}-1}}
$$

uniformly on each compact subset of $\mathbb{C} \backslash[-1,1]$.

Proof. First of all, notice that $\mu^{\prime}>0$ a.e. on $[-1,1]$ implies that $\mu \in \operatorname{Reg}$ (see [12], Theorem 4.1.1). Using (14), (18), and (17), we obtain

$$
\begin{gathered}
\lim _{n \rightarrow \infty} \frac{s_{n, n+k+1}(z)}{s_{n, n+k}(z)}=\lim _{n \rightarrow \infty} \frac{s_{n, n+k+1}(z) g_{n, n+k}(z)}{s_{n, n+k}(z) g_{n, n+k-1}(z)} \times \\
\lim _{n \rightarrow \infty} \frac{p_{n, n+k-1}(z) g_{n, n+k-1}(z)}{p_{n, n+k}(z) g_{n, n+k}(z)} \times \lim _{n \rightarrow \infty} \frac{p_{n, n+k}(z)}{p_{n, n+k-1}(z)}=z+\sqrt{z^{2}-1},
\end{gathered}
$$

and all the limits hold uniformly on each compact subset $K$ of $\mathbb{C} \backslash[-1,1]$. The proof of (19) is complete. From (18) and (14), we immediately obtain (20).

3.3. Strong Asymptotics. Set $\Psi(z)=z+\sqrt{z^{2}-1}$. Let $k$ be a fixed integer. From the results in $[2]^{1}$ it follows that if $\mu \in \mathbf{S}$, then

$$
\lim _{n \rightarrow \infty} \frac{p_{n, n+k}^{2}(z)}{[\Psi(z)]^{2 k-1} w_{n}(z)} B_{n}(z)=\frac{1}{2 \pi}\left[S_{\mu}(\Psi(z))\right]^{2},
$$

uniformly on each compact subset of $\mathbb{C} \backslash[-1,1]$, where

and

$$
B_{n}(z)=\prod_{i=1}^{2 n+1} \frac{\Psi(z)-\Psi\left(w_{n, i}\right)}{1-\overline{\Psi\left(w_{n, i}\right)} \Psi(z)}
$$

$$
S_{\mu}(z)=\exp \left\{\frac{1}{4 \pi} \int_{0}^{2 \pi} \log \left(\mu^{\prime}(\cos \theta)|\sin (\theta)|\right) \frac{e^{i \theta}+z}{e^{i \theta}-z} d \theta\right\}, \quad|z|>1 .
$$

If $w_{n, i}=\infty$ the corresponding factor in $B_{n}$ must be substituted by $1 / \Psi(z)$. Therefore, as a particular case, when $w_{n} \equiv 1$ for all $n \in \mathbb{N}$ one obtains the well known formula

$$
\lim _{n \rightarrow \infty} \frac{p_{n}(z)}{[\Psi(z)]^{n}}=\frac{1}{\sqrt{2 \pi}} S_{\mu}(\Psi(z)),
$$

uniformly on each compact subset of $\mathbb{C} \backslash[-1,1]$.

Using these limit relations and the results above one obtains strong asymptotics for generalized Stieltjes polynomials.

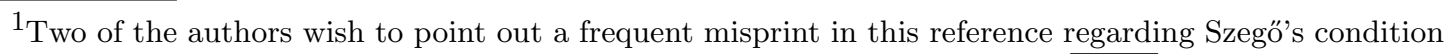
on $[-1,1]$. In particular, it is necessary to substitute $\log \mu^{\prime}(x)$ by $\log \mu^{\prime}(x) / \sqrt{1-x^{2}}$ in the conditions of Theorem 4 and thereafter whenever Szegö's condition is required.
} 
Theorem 5. Suppose that the sequences of polynomials $\left\{w_{n}\right\}$ and $\left\{v_{n}\right\}$ have the same zero asymptotic distribution. Let $k$ be a fixed integer, $k \geq 0$. If $\mu \in \mathbf{S}$ then

$$
\lim _{n \rightarrow \infty} \frac{s_{n, n+k+1}^{2}(z)}{[\Psi(z)]^{2 k-1} w_{n}(z)} B_{n}(z)=\frac{z^{2}-1}{2 \pi} S_{\mu}^{2}(\Psi(z))
$$

uniformly on each compact subset of $\mathbb{C} \backslash[-1,1]$, where $B_{n}(z)$ and $S_{\mu}(z)$ are as in $(21)$. Proof. From (14), (21), and (18), we have

$$
\begin{aligned}
& \lim _{n \rightarrow \infty} \frac{s_{n, n+k+1}^{2}(z)}{[\Psi(z)]^{2 k-1} w_{n}(z)} B_{n}(z)=\lim _{n \rightarrow \infty} s_{n, n+k+1}^{2}(z) g_{n, n+k}^{2}(z) \times \\
& \lim _{n \rightarrow \infty} \frac{p_{n, n+k}^{2}(z)}{[\Psi(z)]^{2 k-1} w_{n}(z)} B_{n}(z) \times \lim _{n \rightarrow \infty} \frac{1}{g_{n, n+k}^{2}(z) p_{n, n+k}^{2}(z)} \\
& =\frac{z^{2}-1}{2 \pi} S_{\mu}^{2}(\Psi(z)),
\end{aligned}
$$

with uniform convergence on any compact subset $K$ of $\mathbb{C} \backslash[-1,1]$, which proves (22).

\section{Applications}

\subsection{Multipoint Padé-Type Approximation. Set}

$$
\widehat{\mu}(z)=c+\int \frac{d \mu(x)}{z-x}, \quad z \in \overline{\mathbb{C}} \backslash S(\mu), \quad c \in \mathbb{R} .
$$

Let $\left\{w_{n}\right\}_{n \in \mathbb{N}}$ and $\left\{v_{n}\right\}_{n \in \mathbb{N}}$ be as above. It is easy to verify that for each $n \in \mathbb{N}$ there exists a unique rational function $R_{n}=L_{n} /\left(Q_{n} p_{n, n}\right)$, where $L_{n}$ and $Q_{n}$ satisfy:

- $\operatorname{deg} Q_{n} \leq n+1, \operatorname{deg} L_{n} \leq 2 n+1$, and $Q_{n} \not \equiv 0$.

- $\frac{Q_{n} p_{n, n} \widehat{\mu}-L_{n}}{w_{n} v_{n}} \in H(\mathbb{C} \backslash S(\mu))$, where $H(\mathbb{C} \backslash S(\mu))$ denotes the set of all holomorphic functions defined on $\mathbb{C} \backslash S(\mu)$.

- $\frac{Q_{n} p_{n, n} \widehat{\mu}-L_{n}}{w_{n} v_{n}}(z)=\mathcal{O}\left(\frac{1}{z^{n+2}}\right), \quad z \rightarrow \infty$.

Hence, by the use of Cauchy and Fubibi Theorems, we obtain

$$
\int x^{k} Q_{n}(x) p_{n, n}(x) \frac{d \mu(x)}{w_{n}(x) v_{n}(x)}=0, \quad k=0,1, \ldots, n .
$$

Thus, taking $Q_{n}$ to be monic we have that $Q_{n} \equiv S_{n, n+1}$. It can also be shown (see, for instance, Lemma 6.1.2 in [12]) that

$$
\widehat{\mu}(z)-R_{n}(z)=\frac{w_{n}(z) v_{n}(z)}{\left(s_{n, n+1} p_{n, n} h\right)(z)} \int \frac{\left(s_{n, n+1} p_{n, n} h\right)(x)}{w_{n}(x) v_{n}(x)} \frac{d \mu(x)}{z-x},
$$

for all $z \in \overline{\mathbb{C}} \backslash S(\mu)$, where $h$ is any polynomial of degree less than or equal to $n+1$.

We are ready for 
Theorem 6. Suppose that the sequences of polynomials $\left\{w_{n}\right\}$ and $\left\{v_{n}\right\}$ have $\nu$ as their zero asymptotic distribution. Let $S(\mu)$ be regular and $\mu \in \mathbf{R e g}$. Then, on each compact subset $K \subset \overline{\mathbb{C}} \backslash E$, we have

$$
\begin{aligned}
& \limsup _{n \rightarrow \infty}\left\|\widehat{\mu}(z)-R_{n}(z)\right\|_{K}^{1 / 3 n} \leq \\
& \left\|\exp \left\{-G_{\Omega}(\nu ; z)\right\}\right\|_{K}\left\|\exp \left\{G_{\Omega}(\nu ; z)\right\}\right\|_{\operatorname{Co}(S(\mu))} .
\end{aligned}
$$

Proof. Fix a compact set $K \subset \mathbb{C} \backslash E$. Let $V$ be a neighbourhood of $\mathbf{L}$ such that $\bar{V} \subset$ $\mathbb{C} \backslash E$. Take $n_{0}$ sufficiently large so that for $n \geq n_{0}$ all the zeros of $v_{n}$ lie in $V$. Fix $r>\left\|G_{\Omega}(\nu ; \zeta)\right\|_{\mathrm{Co}(S(\mu))}$ so that $\bar{V} \cup K$ lies in the unbounded component of $\mathbb{C} \backslash \gamma_{r}$, where $\gamma_{r}=\left\{\zeta \in \mathbb{C}: G_{\Omega}(\nu ; \zeta)=r\right\}$. For short, let us denote $w_{n}(z) v_{n}(z) /\left(s_{n, n+1}^{2}(z) p_{n, n}(z)\right)$ by $h_{n}(z)$. Taking $h \equiv s_{n, n+1}$ in (23), we have that

$$
\widehat{\mu}(z)-R_{n}(z)=h_{n}(z) \int \frac{1}{h_{n}(x)} \frac{d \mu(x)}{z-x}, \quad z \in K .
$$

Since $1 /\left((z-x) h_{n}(x)\right)$ is analytic in an open neighbourhood of the bounded component of $\mathbb{C} \backslash \gamma_{r}$, we may use Cauchy's integral formula and Fubini's Theorem to obtain

$$
\widehat{\mu}(z)-R_{n}(z)=h_{n}(z) \int_{\gamma_{r}} \frac{1}{h_{n}(\zeta)} \frac{\widehat{\mu}(\zeta)}{z-\zeta} d \zeta, \quad z \in K
$$

Hence

$$
\left\|\widehat{\mu}(z)-R_{n}(z)\right\|_{K} \leq C \frac{\left\|h_{n}\right\|_{K}}{\inf _{\zeta \in \gamma_{r}}\left|h_{n}(\zeta)\right|},
$$

where $C$ is a positive constant depending on the length of $\gamma_{r}$ and the distance between $\gamma_{r}$ and $K$, but not on $n \geq n_{0}$. Therefore,

$$
\limsup _{n \rightarrow \infty}\left\|\widehat{\mu}(z)-R_{n}(z)\right\|_{K}^{1 / 3 n} \leq \frac{\limsup _{n \rightarrow \infty}\left\|h_{n}\right\|_{K}^{1 / 3 n}}{\lim _{n \rightarrow \infty} \inf _{\zeta \in \gamma_{r}}\left|h_{n}(\zeta)\right|^{1 / 3 n}} .
$$

From (15), (5), and the uniform convergence of $\left|w_{n} v_{n}\right|^{1 / n}$, we obtain

$$
\lim _{n \rightarrow \infty}\left|h_{n}(z)\right|^{1 / 3 n}=\exp \left\{-G_{\Omega}(\nu, z)\right\},
$$

uniformly on compact subsets of $\mathbb{C} \backslash\{\mathbf{L} \cup E\}$. Using (15), (5), and the Lower Envelope Theorem, we have

$$
\limsup _{n \rightarrow \infty}\left|h_{n}(z)\right|^{1 / 3 n} \leq \exp \left\{-G_{\Omega}(\nu, z)\right\}
$$

uniformly on compact subsets of $\mathbb{C} \backslash E$. By use of $(26)$ and (27), we obtain

$$
\limsup _{n \rightarrow \infty}\left\|h_{n}\right\|_{K}^{1 / 3 n} \leq\left\|\exp \left\{-G_{\Omega}(\nu ; z)\right\}\right\|_{K}
$$

and

$$
\lim _{n \rightarrow \infty} \inf _{\zeta \in \gamma_{r}}\left|h_{n}(\zeta)\right|^{1 / 3 n}=e^{-r}
$$

Relations (28) and (29) together with (25) give

$$
\limsup _{n \rightarrow \infty}\left\|\widehat{\mu}(z)-R_{n}(z)\right\|_{K}^{1 / 3 n} \leq\left\|\exp \left\{-G_{\Omega}(\nu ; z)\right\}\right\|_{K} e^{r} .
$$


The left hand of this inequality does not depend on $r$; therefore, we can make $r$ tend to $\left\|G_{\Omega}(\nu ; \zeta)\right\|_{\mathrm{Co}(S(\mu))}$ obtaining $(24)$ for compact subsets of $\mathbb{C} \backslash E$. Since the function under the norm on the left hand of (24) is analytic in a neighbourhood of infinity, from the Maximum Principle it is obvious that (24) is also true for any $K \subset \overline{\mathbb{C}} \backslash E$.

4.2. Rational Gauss-Kronrod Quadrature. Let us consider the partial fraction decomposition of the approximant $R_{n}$

$$
R_{n}(z)=c+\sum_{i=1}^{N} \sum_{j=0}^{M_{i}} \frac{j ! a_{i, j, n}}{\left(z-z_{n, i}\right)^{j+1}} .
$$

$N$ denotes the total number of distinct poles of $R_{n}$. The points $z_{n, i}$ are the zeros of $s_{n, n+1} p_{n, n}$. Though the zeros of $p_{n, n}$ are simple they may coincide with zeros of $s_{n, n+1}$; therefore, for given $z_{n, i}$ any value of $M_{i}$ is possible. Obviously, $N=N(n)$ and $M_{i}=M_{i}(n)$, but in order to simplify the notation, we omit the explicit reference to this dependence.

Let $f$ be an analytic function on a neighbourhood $V$ of the compact set $E$. Set

$$
I(f)=\int f(x) d \mu(x), \quad I_{2 n+1}^{G K}(f)=\sum_{i=1}^{N} \sum_{j=0}^{M_{i}} a_{i, j, n} f^{(j)}\left(z_{n, i}\right)
$$

and denote $I(f)-I_{2 n+1}^{G K}(f)$ by $E_{2 n+1}^{G K}(f)$. Under the conditions of Theorem 2, from Corollary 1, we know that for $n \geq n_{0}(V)$ all the zeros of $s_{n, n+1}$ are contained in $V$ and the expressions above make sense. In the sequel, we only consider sufficiently large $n$ 's. Notice that if the zeros $z_{n, i}$ are all simple (which is not known in general) we obtain the rational Gauss-Kronrod quadrature rule and if, additionally, $w_{n} \equiv v_{n} \equiv 1, n \in \mathbb{N}$, then $I_{2 n+1}^{G K}$ is the usual Gauss-Kronrod quadrature formula. Even for this classical Gauss-Kronrod quadrature rule little is known about the properties of the zeros and positivity of the coefficients $a_{i, j, n}$ (cf. [4] or the introduction in [1]). From results obtained independently by Peherstorfer [6] and Notaris [5], it follows that $I_{2 n+1}^{G K}$ has simple nodes contained in $\operatorname{Co}(S(\mu))$ and positive coefficients $a_{i, j, n}$, provided that $d \mu(x)=\sqrt{1-x^{2}}, \operatorname{deg} w_{n} \leq n$, and $v_{n} \equiv 1$. We think that it would be worth finding general classes of measures for which rational Gauss-Kronrod quadrature rule verifies the former properties.

Let us consider the degree of exactness of the quadrature formula $I_{2 n+1}^{G K}$.

Lemma 5. There exists $N \in \mathbb{N}$ such that for each $n \geq N$ we have

$$
I\left(\frac{h}{w_{n} v_{n}}\right)=I_{2 n+1}^{G K}\left(\frac{h}{w_{n} v_{n}}\right),
$$

where $h$ is any polynomial of degree less than or equal to $3 n+1$.

Proof. Let $V$ be a neighbourhood of $E$ such that $V \cap \mathbf{L}=\emptyset$. Let $\gamma$ be an analytic Jordan curve such that $V$ lies in the bounded component of $\mathbb{C} \backslash \gamma$ and $\mathbf{L}$ in the unbounded one. For $n \geq N$, all the zeros of $S_{n, n+1}$ belong to $V$ and all of those of $w_{n} v_{n}$ lie in the unbounded component of $\mathbb{C} \backslash \gamma$. From $(23)$ we know that $\left(\widehat{\mu}-R_{n}\right) /\left(w_{n} v_{n}\right)$ is holomorphic in $\overline{\mathbb{C}} \backslash \bar{V}$ and

$$
\frac{\widehat{\mu}-R_{n}}{w_{n} v_{n}}(z)=\mathcal{O}\left(\frac{1}{z^{3 n+3}}\right), \quad z \rightarrow \infty .
$$


Then, if $h$ is any polynomial of degree less than or equal to $3 n+1, h\left(\widehat{\mu}-R_{n}\right) /\left(w_{n} v_{n}\right)$ has a zero at infinity of multiplicity at least two. Therefore, we can use Cauchy's Theorem, Fubini's Theorem, and Cauchy's integral formula to obtain

$$
\begin{gathered}
0=\int_{\gamma} \frac{h\left(\widehat{\mu}-R_{n}\right)}{w_{n} v_{n}}(\zeta) d \zeta=\int_{\gamma} \frac{h(\widehat{\mu}-c)}{w_{n} v_{n}}(\zeta) d \zeta-\int_{\gamma} \frac{h\left(R_{n}-c\right)}{w_{n} v_{n}}(\zeta) d \zeta \\
=2 \pi i\left[\int \frac{h}{w_{n} v_{n}}(x) d \mu(x)-\sum_{i=1}^{N} \sum_{j=0}^{M_{i}} a_{i, j, n}\left(\frac{h}{w_{n} v_{n}}\right)^{(j)}\left(z_{n, i}\right)\right] \\
=2 \pi i\left[I\left(\frac{h}{w_{n} v_{n}}\right)-I_{2 n+1}^{G K}\left(\frac{h}{w_{n} v_{n}}\right)\right], \quad n \geq N .
\end{gathered}
$$

Now, let us prove

Theorem 7. Let $f$ be an analytic function on a simply connected neighbourhood $V$ of $E$. Then, under the conditions of Theorem 6, we have

$$
\limsup _{n \rightarrow \infty}\left|E_{2 n+1}^{G K}(f)\right|^{1 / 3 n} \leq\left\|\exp \left\{-G_{\Omega}(\nu ; z)\right\}\right\|_{\partial V}\left\|\exp \left\{G_{\Omega}(\nu ; z)\right\}\right\|_{\operatorname{Co}(S(\mu))},
$$

where $\partial V$ stands for the set of boundary points of $V$.

Proof. Let $W$ be a neighbourhood of $E$ with $\bar{W} \subset V$. There exists a natural number $n_{0}(W)$ such that for each $n \in \mathbb{N}$ with $n \geq n_{0}(W)$ the polynomial $s_{n, n+1}$ has all its zeros contained in the open set $W$.

Let $\gamma$ be an analytic Jordan curve contained in $V$ such that $W$ lies in the bounded component of $\mathbb{C} \backslash \gamma$. Using similar arguments to those employed in the proof of Lemma 5 , it is easy to verify that

$$
E_{2 n+1}^{G K}(f)=\frac{1}{2 \pi i} \int_{\gamma} f(\zeta)\left(\widehat{\mu}-R_{n}\right)(\zeta) d \zeta .
$$

From this equality and (24), we obtain

$$
\limsup _{n \rightarrow \infty}\left|E_{2 n+1}^{G K}(f)\right|^{1 / 3 n} \leq\left\|\exp \left\{-G_{\Omega}(\nu ; z)\right\}\right\|_{\gamma}\left\|\exp \left\{G_{\Omega}(\nu ; z)\right\}\right\|_{\operatorname{Co}(S(\mu))} .
$$

We can choose $\gamma$ as close to $\partial V$ as we please, so (30) immediately follows.

\section{REFERENCES}

[1] M. Bello Hernández, B. de la Calle Ysern, J. J. Guadalupe Hernández, and G. López Lagomasino, Asymptotics for Stieltjes polynomials, Padé approximants, and Gauss-Kronrod quadrature, J. Anal. Math. 86 (2002), 1-23.

[2] B. De la Calle Ysern and G. López Lagomasino, Strong asymptotics of orthogonal polynomials with varying measures and Hermite-Padé approximants, J. Comput. Appl. Math. 99 (1998), 91-103.

[3] G. López Lagomasino, Asymptotics of polynomials orthogonal with respect to varying measures, Constr. Approx. 5 (1989), 199-219.

[4] G. Monegato, Stieltjes polynomials and related quadrature rules, SIAM Rev. 24 (1982), 137-158.

[5] S. E. Notaris, Gauss-Kronrod quadrature formulae for weight functions of Bernstein-Szegő type. II, J. Comput. Appl. Math. 29 (1990), 161-170. 
[6] F. Peherstorfer, Weight functions admitting repeated positive Kronrod quadrature, BIT 30 (1990), $145-151$.

[7] F. Peherstorfer, On the asymptotic behaviour of functions of second kind and Stieltjes polynomials, and on Gauss-Kronrod quadrature formulas, J. Approx. Theory 70 (1992), 156-190.

[8] F. Peherstorfer and K. Petras, Ultraspherical Gauss-Kronrod quadrature is not possible for $\lambda>3$, SIAM J. Numer. Anal. 37 (2000), 927-948.

[9] E. A. Rakhmanov, On asymptotic properties of polynomials orthogonal on the circle with weights not satisfying Szegö's condition, Math. USSR. Sbornik. 58,1 (1987), 149-167.

[10] T. Ransford, "Potential Theory in the Complex Plane", London Mathematical Society, Student Texts 28, Cambridge University Press, New York, 1995.

[11] E. B. SAFF AND V. Totik, "Logarithmic Potentials with External Fields", A Series of Comprehensive Studies in Math. 316, Springer-Verlag, Berlin, 1997.

[12] H. Stahl and V. Totik, "General Orthogonal Polynomials", Cambridge University Press, Cambridge, 1992.

M. Bello Hernández, Dpto. de Matemáticas y Computación, Universidad de la Rioja, Edificio J. L. Vives, Luis de UlloA S/n, 26004 Logroño, Spain

E-mail address: mbello@dmc.unirioja.es

B. de la Calle Ysern, Dpto. de Matemática Aplicada, E. T. S. De Ingenieros Industriales, Universidad Politécnica de Madrid, José G. Abascal 2, 28006 Madrid, Spain

E-mail address: bcalle@etsii.upm.es

G. López lagomasino, Dpto. de Matemáticas, Escuela Politécnica Superior, Universidad Carlos III de Madrid, Universidad 30, 28911 Leganés, Spain

E-mail address: lago@math.uc3m.es 